\title{
Implementing Lean Quality Improvement in Primary Care: Impact on Efficiency in Performing Common Clinical Tasks
}

\author{
Dorothy Y. Hung, Ph. D(D, Quan A. Truong, M. P. H., and Su-Ying Liang, Ph. D
}

Palo Alto Medical Foundation Research Institute, Sutter Health, Palo Alto, CA, USA.

BACKGROUND: Many primary care practices have adopted Lean techniques to reduce the amount of time spent completing routine tasks. Few studies have evaluated both immediate and sustained impacts of Lean to improve this aspect of primary care work efficiency.

OBJECTIVE: To examine 3-year impacts of Lean implementation on the amount of time taken for physicians to complete common clinical tasks.

DESIGN: Non-randomized stepped wedge with segmented regression and interrupted time series analysis (January 2011-December 2016).

PARTICIPANTS: A total of 317 physician-led teams in 46 primary care departments in a large ambulatory care delivery system.

INTERVENTION: Lean redesign was initiated in one pilot site followed by system-wide spread across all primary care departments. Redesigns included standardization of exam room equipment and supplies, streamlining of call management processes, care team co-location, and team management of the electronic inbox.

MEASURES: Time-stamped EHR tracking of physicians' completion time for 4 common tasks: (1) office visit documentation and closure of patient charts; (2) telephone call resolution; (3) prescription refill renewal; and (4) response to electronic patient messages.

RESULTS: After Lean implementation, we found decreases in the amount of time to complete: office visit documentation (- 29.2\% [95\% CI: -44.2 , - 10.1]), telephone resolution ( $-22.2 \%$ [95\% CI: $-38.1,-2.27])$, and renewal of prescription refills ( $-2.96 \%$ per month [95\% CI: $-4.21,-1.78])$. These decreases were sustained over several years. Response time to electronic patient messages did not change significantly.

CONCLUSIONS: Lean redesigns led to improvements in timely completion of 3 out of 4 common clinical tasks. Our findings support the use of Lean techniques to engage teams in routine aspects of patient care. More research is warranted to understand the mechanisms by which Lean promotes quality improvement and effectiveness of care team workflows.

KEY WORDS: Lean management; primary care redesign; workflow/task completion; physician efficiency; primary care teams.

Preliminary findings based on this research were presented at professional conferences, including annual meetings hosted by AcademyHealth (June 2018) and the Health Care Systems Research Network (April 2019). Received July 29, 2019

Accepted October 13, 2020

Published online November 24, 2020
J Gen Intern Med 36(2):274-9

DOI: $10.1007 / \mathrm{s} 11606-020-06317-9$

(c) Society of General Internal Medicine 2020

\section{INTRODUCTION}

Workflow interventions are increasingly being used to improve efficiency in primary care. ${ }^{1}$ An example of this includes Lean process improvement, which aims to optimize daily workflows by minimizing waste and maximizing valueadded activities. ${ }^{2,3}$ Drawing on approaches originally developed in auto manufacturing, Lean has been widely adopted in health care as a way to optimize the delivery of health services. Positive outcomes of such intervention are observed in a variety of health settings. These outcomes include greater physician productivity and staff satisfaction, ${ }^{4-7}$ decreased hospital length of stay, ${ }^{8}$ and reduced laboratory errors and turnaround time for pathology reports. ${ }^{9-11}$

Most research to date focuses on the initial, or immediate, impacts of Lean intervention. Few longitudinal studies have been conducted over time to understand longer-term effects of Lean redesigns, ${ }^{3,12}$ particularly on work efficiency regarding timely task completion among primary care physicians. Monitoring both initial impacts, as well as sustained trends, is critical to understand the full result of performance improvement efforts. Potential for "backsliding" or relapse to the status quo $^{13}$ is especially relevant in primary care settings where new initiatives are frequently introduced. As Lean continues to be adopted in primary care settings, more evidence of both its immediate and sustained effect is needed.

We examined the impact of Lean workflow redesigns on the amount of time that primary care physicians required to complete common clinical tasks. Executive leaders envisioned the Lean initiative as a system-wide transformation focused on optimizing clinical work spaces and care team processes. Improved task efficiency was conceived as reduced waste in completing daily activities, leading to quicker turnaround time for common clinical tasks. For example, co-locating physician and medical assistant care teams was intended to enhance efficiency of communication, while streamlining call management functions aimed to facilitate more rapid call resolution. In this study, we evaluated the impact of Lean workflow redesigns on the amount of time taken by primary care physicians to complete routine tasks. We expected that Lean would result in quicker turnaround time for four common activities (office 
visit documentation and chart closure, telephone call resolution, prescription refill renewal, and response to electronic patient messages).

\section{METHODS}

\section{Overview}

The Lean implementation and evaluation followed a nonrandomized, stepped wedge design. In total, 46 primary care departments housed in 17 ambulatory clinic facilities implemented Lean workflow redesigns. We measured the amount of time to complete key tasks at baseline prior to intervention, and as compared with values measured at 1,2, and 3 or more years after Lean implementation. System-wide introduction of Lean redesigns occurred in three phases. Phase 1 involved all primary care departments in one selected pilot clinic, phase 2 included all primary care departments in three "beta" test clinics, and phase 3 entailed the spread of Lean workflows across all primary care departments in 13 remaining primary care clinics across the system (see Fig. 1). For this study, our total observation period spanned 6 years, during which we continued to monitor data for 3 years after the spread of redesigns. This study was approved by the Institutional Review Board at the Palo Alto Medical Foundation.

\section{Study Sample}

The study included 317 primary care physicians and clinical staff teams in 46 internal medicine, family medicine, and pediatric departments in a large ambulatory care system. This system was the product of a recent merger of three separate medical groups into a single entity, and Lean was the first large quality improvement initiative undertaken by the newly consolidated organization. To minimize the effects of turnover during the study period, analyses were based on physicians who were (1) continuously employed more than 5\% FTE for at least half of the time periods both before and after Lean workflows were introduced at each clinical site and (2) employed for at least 6 months during the pre-Lean period and at least 12 months during the post-Lean period.

\section{Intervention}

Executive leaders with the support of a newly created "Lean Promotion Office" (LPO) introduced the redesigns as a system-wide strategic initiative beginning in primary care. These redesigns sought to increase work efficiency and functioning among physicians and staff, while also improving service quality experienced by patients. Lean intervention formally began with improving work environments and care team processes. Hired Lean consultants and internal LPO trainers consisting of local operational leaders and physician champions worked with frontline physicians and staff to standardize patient exam rooms, clinical work spaces, and daily workflows. No additional staff or IT resources were provided by the initiative beyond available capabilities. At each site, workshops were held by current system leaders, Lean consultants, and LPO trainers to introduce the newly designed standards. This was followed by a 3-month transitional period of

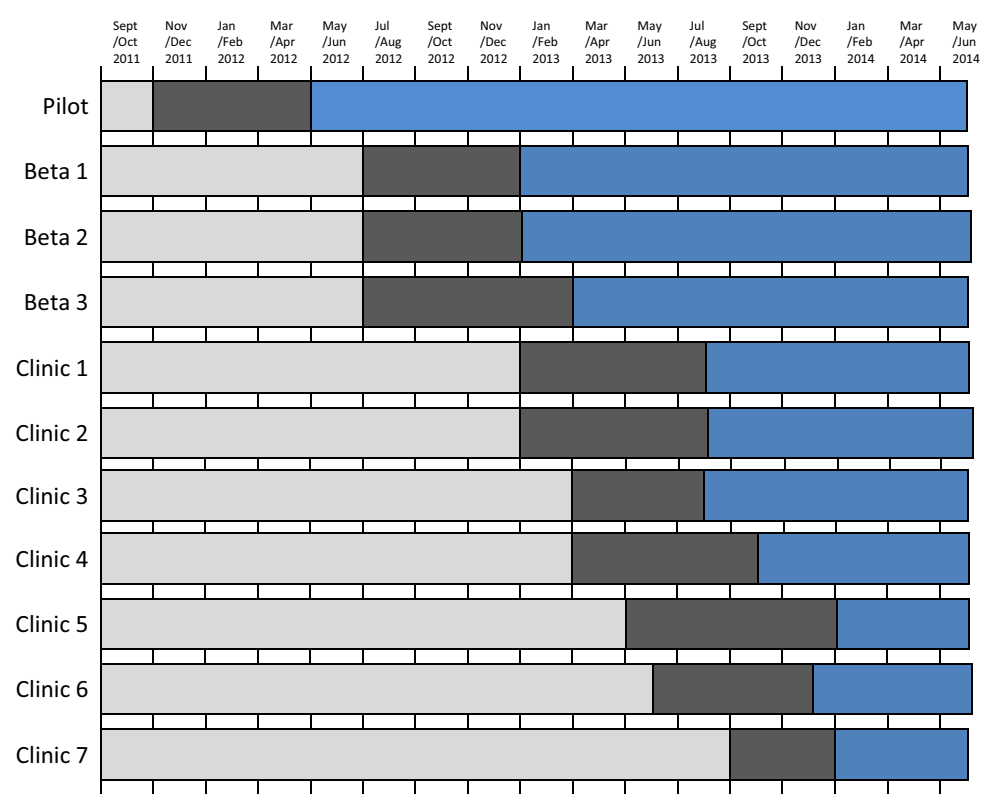

Note: All Clinic facilities (except for 4 and 7) have additional satellite sites that were included for analysis.

Pre-Lean period

Training/Implementation period

Post-Lean period (forward until Dec. 2016)

Figure 1 Non-randomized stepped wedge design of Lean implementation. 
learning and stabilization using visual dashboards in each department.

Lean redesigns included (1) " $5 \mathrm{~S}$ " standardization of medical equipment, supplies, and health education materials in all patient exam rooms; (2) streamlining of call management and call center functions; (3) co-location of physician and medical assistant (MA) care teams in a shared workspace; and (4) redesign of daily care team workflows. These new workflows included morning huddles to review patient schedules; agenda setting with patients at the start of each office visit; and joint management of electronic in-baskets by both the physician and MA. In this capacity, MAs helped manage and triage items in the physician's electronic inbox, which included patient messages, prescription refills, lab/imaging results, and referral requests. Improvements made to both the organization of clinical spaces and care team workflows aimed to increase overall efficiencies among primary care physicians (e.g., less time spent searching for materials and supplies, more timely communication with care team members between patient visits). Taken as a whole, these changes were intended to facilitate quicker completion of routine clinical tasks throughout the day.

\section{Data Sources and Measures}

Data were sourced from the organization's time-stamped electronic health record (EHR) system that tracks physician activities. We measured time to completion of four key tasks revealing potential effects of Lean on physician work time. For all physicians, we measured average completion times monthly at baseline, compared with average completion times going forward in the following 3 years. We defined maintenance of effects as a lack of significant change in values from the first year of Lean intervention to the third year of followup. Our measures were the amounts of time taken to (1) complete documentation of the office visit, measured via EHR time stamp for the end of a patient visit until chart closure; (2) resolve patient telephone calls, measured using the EHR time stamp on an incoming call until the closing of the physician telephone note; (3) renew prescription medication refills, measured as the EHR time stamp for a patient's request for prescription renewal to the time the physician ordered or denied the request; and (4) respond to patients' electronic messages, based on the EHR time stamp for message arrival in the physician's inbox to the closing of the online encounter.

\section{Statistical Analysis}

We used segmented regression with interrupted time series analysis to assess both the immediate and sustained impact of Lean redesigns on task completion time. This analytic approach was chosen to account for the phased implementation of Lean while also adjusting for secular trends. ${ }^{14-16}$ The data were analyzed using a non-randomized stepped wedge study design with one-way crossover. ${ }^{17,18}$ Main effects included coefficients reflecting both immediate impacts of Lean (represented by model intercepts comparing baseline and post-Lean changes) and gradual impacts over time (slopes measuring changes in outcomes per month) for three consecutive periods of observation. Leveraging the segmented regression approach, these three periods included the first, second, and third year and beyond of Lean implementation in each primary care site. This approach also allowed us to assess whether initial impacts were maintained over time by comparing first-year results specifically to those observed in subsequent time periods. After obtaining model estimates, coefficients were log transformed into percent changes in outcomes: $\beta_{\text {it }}=\log Y_{2}-\log Y_{1}$ or $\log \left(Y_{2} / Y_{1}\right)$; then $Y_{2} /$ $\mathrm{Y}_{1}=\exp \left(\beta_{\mathrm{it}}\right) ; \mathrm{Y}_{2}=\exp \left(\beta_{\mathrm{it}}\right) \mathrm{Y} 1 ; \%$ change $=1-\exp \left(\beta_{\mathrm{it}}\right)$.

In all models, we adjusted for secular trends and potential confounders across physicians in each department. These included scheduled clinic hours, mean age of patients on a panel, physician demographics, measures of workload (e.g., number of office visits, phone calls, electronic messages), productivity (e.g., wRVUs generated per visit), and proportion of new patient visits per month. To account for site-level effects and the nested structure of physicians within clinical departments, we included unique department indicators as random effects. ${ }^{19}$ This approach allowed Lean effects to vary by site (i.e., impact of Lean was not assumed to be the same, or fixed, in each site). We accounted for autocorrelation of repeated measures over time using a first-order autoregressive $\mathrm{R}$-side covariance structure.

\section{RESULTS}

Table 1 presents a description of all study sites. The 46 primary care departments were evenly represented by internal medicine, family medicine, and pediatrics. The average practice size was approximately 20 members with a 1.5 staff-tophysician ratio. The average number of months post-Lean implementation in each site was 43.6 months, or roughly 3.5 years. At baseline prior to Lean redesign, the average time for physicians to document and close patient charts after office visits was $4 \mathrm{~h}$ and $45 \mathrm{~min}$. The average phone resolution time was $4 \mathrm{~h}$ and $4 \mathrm{~min}$, while physician response to electronic messages was $4 \mathrm{~h}$ and $15 \mathrm{~min}$. The average time to renew prescription refills was $9 \mathrm{~h}$ and $33 \mathrm{~min}$. After Lean implementation, changes in baseline metrics were most pronounced in two of the four study measures: documentation and closure of patient charts after the office visit, and resolution of telephone calls. These changes persisted 3 years after Lean was introduced in study sites.

Table 2 displays segmented regression results for office visit chart closure times. We found significant improvements in this metric during the first year of Lean redesigns. Specifically, the amount of time taken to complete documentation and close patient charts after the office visit decreased significantly from baseline by $29.2 \%$ (95\% CI: $-44.2,-10.1)$. This improvement was maintained several years later, as indicated 
Table 1 Description of Site Characteristics $(N=46$ Primary Care Departments)

\begin{tabular}{|c|c|c|}
\hline & $\begin{array}{l}\text { Mean } \\
(\text { or } N)\end{array}$ & $\begin{array}{l}\text { SD } \\
(\text { or } \%)\end{array}$ \\
\hline \multicolumn{3}{|l|}{ Department type } \\
\hline Internal medicine & $(15)$ & $(32.6 \%)$ \\
\hline Family medicine & (16) & $(34.8 \%)$ \\
\hline Pediatrics & $(15)$ & $(32.6 \%)$ \\
\hline Practice size (FTE) & 19.7 & 2.65 \\
\hline Staff:physician ratio & 1.5 & 0.62 \\
\hline Study months post-lean redesigns & 43.6 & 1.41 \\
\hline \multicolumn{3}{|l|}{ Office visit chart closure timet } \\
\hline Baseline (pre-Lean) & 4.8 & 8.7 \\
\hline 1 year post-Lean & 3.2 & 5.8 \\
\hline 2 years post-Lean & 3.5 & 8.9 \\
\hline 3 years post-Lean & 3.7 & 8.1 \\
\hline \multicolumn{3}{|l|}{ Telephone call resolution timet } \\
\hline Baseline (pre-Lean) & 4.0 & 4.4 \\
\hline 1 year post & 3.3 & 4.1 \\
\hline 2 years post & 3.5 & 3.5 \\
\hline 3 years post & 3.3 & 4.1 \\
\hline \multicolumn{3}{|l|}{ Prescription refill renewal time ${ }^{\dagger}$} \\
\hline Baseline (pre-Lean) & 9.5 & 14.6 \\
\hline 1 year post & 8.8 & 14.9 \\
\hline 2 years post & 8.2 & 8.1 \\
\hline 3 years post & 10.7 & 11.6 \\
\hline \multicolumn{3}{|c|}{ Response time for electronic messages $\dagger$} \\
\hline Baseline (pre-Lean) & 4.3 & 3.3 \\
\hline 1 year post & 4.1 & 3.0 \\
\hline 2 years post & 3.3 & 2.4 \\
\hline 3 years post & 3.6 & 2.7 \\
\hline
\end{tabular}

FTE, full-time equivalent

Univariate, unadjusted times in average number of hours

by a lack of significant difference in results between the first and subsequent years of Lean implementation.

Table 3 shows similar findings for telephone resolution times. Compared with baseline levels, the amount of time taken to address telephoned patient care items decreased by $22.2 \%$ during the first year of Lean implementation $(95 \%$ CI: $-38.1,-2.27)$. This improvement in resolution time persisted three years later. As shown in Table 4, although the change was less dramatic, we also detected a positive effect on the amount of time taken for physicians to address prescription refill requests. Compared with trends observed in the baseline period, there was a $2.96 \%$ gradual decrease over time, i.e., measured by the slope, during the first year of Lean redesign (95\% CI: $-4.21,-1.78)$. In other words, there were incre-

Table 2 Office Visit Chart Closures*

\begin{tabular}{llll}
\hline \hline & $\begin{array}{l}\text { Coefficient } \\
\text { (Log) }\end{array}$ & $\begin{array}{l}\text { \% } \\
\text { Change }^{\dagger}\end{array}$ & 95\% CI \\
\hline $\begin{array}{l}\text { Baseline (pre- } \\
\text { lean) }\end{array}$ & 9.41 & - & - \\
1st year post-lean & -0.35 & $-29.2 \%$ & $(-44.2,-10.1)$ \\
2nd year post-lean & -0.19 & $-17.5 \%$ & $(-32.4,3.6)$ \\
3rd year post-lean & -0.22 & $-20.0 \%$ & $(-37.2,2.0)$ \\
\hline
\end{tabular}

*Adjusted for physician scheduled clinic hours, the mean age of patients on a physician's panel, physician demographics, workload and productivity, and proportion of new patient visits. $N=317$ physicians, 46 primary care departments. ${ }^{\dagger}$ Reference groups: Baseline (pre-Lean) for 1st year post-Lean effect; 1st year (initial effect) for both 2nd and $3 r d$ year post-Lean results
Table 3 Telephone Call Resolution*

\begin{tabular}{llll}
\hline \hline & $\begin{array}{l}\text { Coefficient } \\
\text { (Log) }\end{array}$ & \% Change & \\
& 95\% CI \\
\hline Baseline (pre-Lean) & 9.730 & - & - \\
1st year post-Lean & -0.251 & $-22.2 \%$ & $(-38.1,-2.3)$ \\
2nd year post-Lean & -0.102 & $-9.70 \%$ & $(-21.7,4.1)$ \\
3rd year post-Lean & -0.048 & $-4.69 \%$ & $(-14.3,5.9)$ \\
\hline
\end{tabular}

*Adjusted for physician scheduled clinic hours, the mean age of patients on a physician's panel, physician demographics, workload and productivity, and proportion of new patient visits. $N=317$ physicians, 46 primary care departments. ${ }^{\dagger}$ Reference groups: Baseline (pre-Lean) for 1st year post-Lean effect; 1st year (initial effect) for both 2 nd and $3 \mathrm{rd}$ year post-Lean results

mental decreases of nearly $3 \%$ per month in the amount of time taken to process prescription refill requests during the first year of Lean implementation.

Finally, Table 5 shows no immediate effect on physician response time to electronic patient messages. Although there appeared to be slight improvements in subsequent post-Lean periods, these results did not reach statistical significance.

\section{DISCUSSION}

Primary care has faced and continues to wrestle with many challenges. Prevailing areas of need include optimizing of practice efficiency and alleviating burnout among the workforce. Lean redesign of care team workflows and other similar interventions may offer a way forward. While such interventions have often demonstrated positive results, ${ }^{7,20-22}$ longerterm effects remain unclear. We found that physician time taken to complete daily tasks improved as a result of Lean redesigns, both after its initial introduction and in subsequent years. Our findings suggest that Lean can yield benefits to both physicians and patients not only by saving physicians time during a typical workday but also by improving service quality through quicker response to patient requests. This finding is complementary to a recent study that found associations between Lean maturity and perceptions of better staff caregiving as an indicator of quality care. ${ }^{12}$

An outstanding challenge in clinical practice is the substantial amount of "desktop medicine" required for managing patient care and handling administrative tasks. According to

Table 4 Prescription Refill Renewals*

\begin{tabular}{llll}
\hline \hline & $\begin{array}{l}\text { Coefficient } \\
\text { (Log) }\end{array}$ & \% Change $^{\dagger}$ & 95\% CI \\
\hline Baseline (pre-Lean) & 9.733 & \multicolumn{1}{c}{} & \\
1 st year post-Lean & $-0.148^{\star}$ & $-13.8 \%$ & - \\
2nd year post-Lean & -0.054 & $-5.26 \%$ & $(-18.5,1.2)$ \\
3rd year post-Lean & 0.007 & $0.7 \%$ & $(-11.0,14.1)$ \\
\hline
\end{tabular}

*Adjusted for physician scheduled clinic hours, the mean age of patients on a physician's panel, physician demographics, workload and productivity, and proportion of new patient visits. $N=317$ physicians, 46 primary care departments. ${ }^{\dagger}$ Reference groups: Baseline (pre-Lean) for 1st year post-Lean effect; 1st year (initial effect) for both 2nd and $3 \mathrm{rd}$ year post-Lean results. ${ }^{7}$ Slope (monthly change) was $-3.0 \%(95 \%$ CI: $-4.2,-1.8)$ 
Table 5 Response to Electronic Patient Messages*

\begin{tabular}{llll}
\hline \hline & $\begin{array}{l}\text { Coefficient } \\
\text { (Log) }\end{array}$ & \% Change ${ }^{\dagger}$ & 95\% CI \\
\hline Baseline (pre-Lean) & 9.838 & - & - \\
1st year post-Lean & -0.103 & $-9.8 \%$ & $(-21.7,3.8)$ \\
2nd year post-Lean & -0.109 & $-10.3 \%$ & $(-21.2,2.1)$ \\
3rd year post-Lean & -0.058 & $-5.6 \%$ & $(-14.9,4.6)$ \\
\hline *Adjusted for physician scheduled clinic hours, the mean age of patients \\
on a physician's panel, physician demographics, workload and \\
productivity, and proportion of new patient visits. N= 317 physicians, \\
46 primary care departments. "Reference groups: Baseline (pre-Lean) \\
for 1st year post-Lean effect; 1st year (initial effect) for both 2nd and \\
3rd year post-Lean results
\end{tabular}

a number of studies on physician work, nearly one-third to one-half of an average workday is spent on activities that occur outside the exam room. ${ }^{23-25}$ This predominantly involves documentation of office visits, follow-up on patient care items, and addressing billing requirements. Physicians report that a significant number of follow-up and task coordination outside the exam room could be performed by clinical staff. ${ }^{25}$ By engaging care team members and reallocating responsibilities where appropriate, Lean aims to achieve a more efficient distribution of labor to maximize available support and resources. As each member works "to the top of their license," services are delivered more effectively with full engagement from the care team.

Thus, we assessed whether Lean redesigns were associated with the achievement of task efficiencies outside the exam room. We observed a nearly $30 \%$ improvement in physicians' documentation and closure of patient charts after office visits, and a more than $20 \%$ decrease in telephone resolution time. There was also a small but significant decrease in the amount of time taken to address prescription refill requests. As charting and follow-up care items constitute the highest percentage of time spent outside of office visits, ${ }^{24,25}$ these findings have important implications for daily workdays among overstretched primary care physicians. For example, less time spent on administrative and non-patient facing items could translate to either additional hours of direct patient care per week, or simply shorter clinic days. ${ }^{26}$

We found that Lean redesigns were associated not only with quicker completion of daily tasks in the short-run, but persisted several years later. The positive effects may be suggestive of longer-term impacts of work reallocation and role change among primary care teams. It should be noted that while certain changes, such as reorientation of physical workspace (e.g., 5S, care team co-location), can be uniformly achieved across all sites, other changes involving care team protocols may be more variable depending on unique practice features or implementation processes in each setting. ${ }^{27-29}$ Such influences of organizational context on practice transformation are noted in other studies, including a recent longitudinal study on burnout among care team members. ${ }^{30}$

Of the examined metrics, we did not find significant improvements in the amount of time taken for physicians to respond to electronic patient messages. A possible explanation for this finding may involve not only variability in adoption by care teams but also the substantial increase in patient emails that occurred during our observation period. A new EHR patient portal that allowed patients access to free electronic messaging with their physicians was launched across the health system approximately 1 year prior to the introduction of Lean workflow redesigns. Increasing familiarity with this new service resulted in higher volumes of patient emails over the years. Although not statistically significant, we did observe slight post-Lean improvements in physician response time when compared with baseline trends, which may be suggestive of some mitigating effects of enhanced workflow on the rising number of electronic messages.

A primary limitation of our study is that we cannot determine with certainty the extent to which observed changes were due to Lean intervention itself or to other unmeasured factors. Our use of a non-randomized stepped wedge design as a strong quasi-experimental approach does not enable comparison of Lean with other possible quality improvement methods or with no intervention at all. Our design does serve to adjust for secular trends while allowing for within-site comparisons before and after Lean implementation with comparisons aggregated to the system level. Although the sample size of 46 primary care departments in 17 clinic facilities is robust, the health system's implementation time period limits the ability to use sites not yet intervened upon as contemporaneous controls. Another study limitation is that we studied only the effects of Lean as a whole, and not specific components of Lean redesigns. Finally, while we found significant reductions in time to completion for important primary care tasks, we did not directly assess whether providers or teams experienced less burden.

\section{CONCLUSION}

The ability of physicians to achieve time savings on daily tasks has potential to serve multiple purposes. Quicker completion times can improve the quality of a physician's work life by creating the option of shorter clinic days, or alternatively, more opportunities for patient interaction during scheduled office visits. Importantly, quicker completion of tasks can improve service quality, which may lead to greater patient satisfaction and access to care. As noted by others, understanding patient experiences as a result of Lean intervention is an important outcome and area for further research. ${ }^{12}$ Additionally, more research on the specific mechanisms by which staff can support physician practice will add further insights into the effectiveness of care team workflows. Higher efficiency, quality, and performance are needed to address growing patient demand amidst prevailing financial challenges in primary care. ${ }^{31}$ Work redesigns that optimize the capacity of primary care teams can aid physicians in recapturing time to engage more effectively in delivering patient care. 
Acknowledgments: The authors would like to thank Gianyun Zhang, M.A., and Hal Luft, Ph.D., for early analytic support and substantive feedback on this work.

Corresponding Author: Dorothy Y. Hung, Ph. D; Palo Alto Medical Foundation Research Institute, Sutter Health, Palo Alto, CA, USA (e-mail: hungd@sutterhealth.org).

Funding This study was funded by the Agency for Healthcare Research and Quality grant 5RO1HSO24529.

\section{Compliance with Ethical Standards:}

This study was approved by the Institutional Review Board at the Palo Alto Medical Foundation.

Conflict of Interest: The authors declare that they do not have a conflict of interest.

\section{REFERENCES}

1. Friedman A, Hahn KA, Etz R, et al. A typology of primary care workforce innovations in the United States since 2000. Med Care 2014;52(2):101111.

2. Mazzocato P, Savage C, Brommels M, Aronsson H, Thor J. Lean thinking in healthcare: a realist review of the literature. Qual Saf Health Care 2010;19(5):376-382.

3. D'Andreamatteo A, Ianni L, Lega F, Sargiacomo M. Lean in healthcare: a comprehensive review. Health Policy 2015;119(9):1197-1209.

4. Raab SS, Grzybicki DM, Condel JL, et al. Effect of Lean method implementation in the histopathology section of an anatomical pathology laboratory. J Clin Pathol 2008;61(11):1193-1199.

5. Dickson EW, Anguelov $Z$, Bott $\mathbf{P}$, Nugent A, Walz D, Singh $\mathbf{S}$. The sustainable improvement of patient flow in an emergency treatment centre using Lean. Int J Six Sigma Comp Adv 2008;4(3):289-304.

6. Vats A, Goin KH, Fortenberry JD. Lean analysis of a pediatric intensive care unit physician group rounding process to identify inefficiencies and opportunities for improvement. Pediatr Crit Care Med 2011;12(4):415421.

7. Sullivan P, Soefje S, Reinhart D, McGeary C, Cabie ED. Using Lean methodology to improve productivity in a hospital oncology pharmacy. Am J Health Syst Pharm 2014;71(17):1491-1498.

8. Ng D, Vail G, Thomas S. Applying the Lean principles of the Toyota Production System to reduce wait times in the emergency department. J Can Assoc Emerg Phys 2010;12(1):50-57.

9. Kim CS, Spahlinger DA, Kin JM, Billi JE. Lean health care: what can hospitals learn from a world-class automaker? J Hosp Med 2006; 1(3):191-199.

10. Chassin R. The Six Sigma Initiative at Mount Sinai Medical Center. Mt Sinai J Med 2008;75(1):45-52.

11. Panning $\mathbf{R}$. Using data to make decisions and drive results: a LEAN implementation strategy. Clin Leadersh Manag Rev 2005;19(2):E4.

12. Kaltenbrunner M, Bengtsson L, Mathiassen S, Hogberg H, Engstrom M. Staff perception of Lean, care-giving, thriving and exhaustion: a longitudinal study in primary care. BMC Health Serv Res 2019;19:652671.

13. Bowman CC, Sobo EJ, Asch SM, Gifford AL, Initiative HIHQER. Measuring persistence of implementation: QUERI Series. Implement Sci 2008;3:21.
14. Wagner AK, Soumerai SB, Zhang F, Ross-Degnan D. Segmented regression analysis of interrupted time series studies in medication use research. J Clin Pharm Ther 2002;27(4):299-309.

15. Gebski V, Ellingson K, Edwards J, Jernigan J, Kleinbaum D. Modelling interrupted time series to evaluate prevention and control of infection in healthcare. Epidemiol Infect 2012;140(12):2131-2141.

16. Penfold RB, Zhang F. Use of interrupted time series analysis in evaluating health care quality improvements. Acad Pediatr 2013;13(6 Suppl):S38-44.

17. Hu Y. Stepped wedge, natural experiments and interrupted time series analysis designs. In: Richards DA, Hallsberg I, eds. Complex Interventions in Health: An Overview of Research Methods. London and New York: Routledge Taylor \& Francis Group; 2015.

18. Highfield L, Rajan SS, Valerio MA, Fernandez ME, Bartholomew LK. A non-randomized controlled stepped wedge trial to evaluate the effectiveness of a multi-level mammography intervention in improving appointment adherence in underserved women. Implement Sci. 2015;10(143).

19. Schall R. Estimation in generalized linear models with random effects. Biometrika. 1991;78(4):719-727.

20. Jimmerson C, Weber D, Sobek DK. Reducing waste and errors: piloting lean principles at Intermountain Healthcare. Jt Comm J Qual Patient Saf 2005;31(5):249-257.

21. Kim CS, Spahlinger DA, Billi JE. Creating value in health care: the case for Lean thinking. JCOM. 2009; 16(12):557-562.

22. Hung DY, Harrison MI, Martinez MC, Luft HS. Scaling Lean in primary care: impacts on system performance. Am J Manag Care 2017;23(3):161168.

23. Gottschalk A, Flocke SA. Time spent in face-to-face patient care and work outside the examination room. Ann Fam Med 2005;3(6):488-493.

24. Gilchrist V, McCord G, Schrop SL, et al. Physician activities during time out of the examination room. Ann Fam Med 2005;3(6):494-499.

25. Chen M, Hollenberg $\mathbf{J}$, Michelen W, Peterson $\mathbf{J}$, Casalino L. Patient care outside of office visits: a primary care physician time study. J Gen Intern Med 2011;26(1):58-63.

26. Farber J, Siu A, Bloom P. How much time do physicians spend providing care outside of office visits? Ann Intern Med 2007;147(10):693-698.

27. Hung D, Martinez M, Yakir M, Gray C. Implementing a lean management system in primary care: facilitators and barriers from the front lines. Qual Manag Health Care 2015;24(3):103-108.

28. Hung D, Gray C, Martinez M, Schmittdiel J, Harrison MI. Acceptance of lean redesigns in primary care: A contextual analysis. Health Care Manag Rev 2017;42(3):203-212.

29. Hung DY, Harrison MI, Liang S-Y, Truong QA. Contextual Conditions and Performance Improvement in Primary Care. Qual Manag Health Care 2019;28(2):70-77.

30. Grumbach $\mathbf{K}$, Knox $\mathbf{M}$, Huang B, Hammer $\mathbf{H}$, Kivlahan C, WillardGrace R. A longitudinal study of trends in burnout during primary care transformation. Ann Fam Med 2019;17(S1):9-15.

31. Carayon P, Wetterneck TB, Rivera-Rodriguez AJ, et al. Human factors systems approach to healthcare quality and patient safety. Appl Ergon 2014:45(1):14-25.

Publisher's Note Springer Nature remains neutral with regard to jurisdictional claims in published maps and institutional affiliations. 